\title{
Los recursos tecnológicos como soporte para la enseñanza de las ciencias naturales \\ Technological resources as support in natural sciences teaching
}

\author{
Milagros Esperanza Rojas Salgado ${ }^{1}$ \\ Universidad Nacional Mayor de San Marcos, Perú
}

Recibido: 14-02-2017

Aceptado: 23-05-2017

\section{Cita Recomendada}

Rojas, M. (2017) Los recursos tecnológicos como soporte para la enseńanza de las ciencias naturales. Hamut'ay, 4 (1), 85-95. Recuperado de: http://dx.doi.org/10.21503/hamu.v4i1.1403

\section{RESUMEN}

En este artículo se presenta un estudio etnográfico de la situación actual de la enseñanza de las ciencias naturales en nuestro país, desde la perspectiva de los lineamientos educativos a nivel nacional. Asimismo, se sustenta la necesidad de la integración de los recursos tecnológicos en esta área. El objetivo fue documentar las formas en las que se podría innovar teniendo en cuenta que las Tecnologías de la Información y la Comunicación (TIC) son un medio para alcanzar los resultados de aprendizajes planteados y no un fin en sí mismo, pues permiten aprovechar las competencias que nuestros estudiantes están desarrollando en su día a día para lograr y/o empoderar sus aprendizajes. A partir del estudio se puede concluir que la integración de las TIC en la enseñanza de las Ciencias Naturales es necesaria y pertinente, dado que contribuyen al logro de los resultados de aprendizaje esperados en los estudiantes, generando competencias no solo a nivel cognitivo sino también a nivel actitudinal.

Palabras Clave: Enseñanza de las Ciencias Naturales, recursos tecnológicos, aprendizajes.

\section{Abstract}

This article presents an ethnographic study of the current situation of Natural Sciences teaching in our country, from the perspective of the national education guidelines. It also highlights the need to integrate technological resources in this area. The objective was to document the ways in which innovations could be made, taking into account that Information and Communication Technologies (ICTs) are a means to achieve the desirable learning outcomes and not an end in itself; since they allow us to benefit from the competences that our students develop in their daily basis to achieve and / or empower their learning. From the study it can be concluded that the integration of ICTs in Natural Sciences teaching is necessary and relevant, since they contribute to the achievement of

\footnotetext{
1 Licenciada en Educación Secundaria de la Universidad Nacional Mayor de San Marcos, especialidad de Biología y Química, Licenciada en Psicología Humana de la Universidad Alas Peruanas. Candidata a magister en Gestión de la Educación y Doctorando en Educación por la Universidad Inca Garcilaso de la Vega. Con estudios de Post grado en Neuropedagogía. E-mail: milagrosrojassalgado@ gmail.com
} 
the expected learning outcomes in the students, generating competences not only at the cognitive but also at attitudinal level.

Keywords: Natural Sciences teaching, technological resources, learning.

\section{INTRODUCCIÓN}

A menudo se habla de la implementación de las TIC como una necesidad primordial en las escuelas. El hecho que el servicio educativo que se brinda esté en completa consonancia con los estudiantes que lo reciben, considerando la sociedad en la que estos se desenvuelven, se ha destacado como una de esas prioridades que se deben de atender desde las políticas y normativas que brinda nuestro sistema educativo nacional, hasta su consecuente implementación, seguimiento, análisis de resultados e innovaciones respectivas. Sin embargo, la escuela actual en Perú todavía se encuentra en estado incipiente respecto a los espacios que brinda para que los estudiantes cuenten con las posibilidades de usar recursos tecnológicos de forma simultánea. Por el contrario, la participación en clases, en la fase de recuperación de aprendizajes previos, aún se realiza en actividades plenarias durante los diez o quince minutos iniciales, para que cinco de los más de veinte estudiantes brinden su punto de vista sobre un tema. ¿Y los demás?, ¿quedarán para la próxima clase? Es más: todos los estudiantes deben de participar sobre la misma "situación significativa". Cabe preguntarse entonces: ¿cómo podrían gestionar sus aprendizajes si aún no pueden ser autónomos en lo que les genera interés? ¿cómo podrán los docentes identificar las necesidades de aprendizaje de sus estudiantes si estos no han tenido la oportunidad de poner en evidencia sus concepciones alternativas sobre las cuales se tendría que trabajar? Puede ser que también valga la pena preguntarse ¿por qué necesariamente se debe cambiar el modelo de escuela del siglo XX para este siglo. ¿Funcionaba mal la escuela del siglo pasado? La respuesta es rotunda; evidencias sobran para sustentar el desfase entre lo que se hace en la escuela y lo que requiere la sociedad, donde todo gira básicamente en torno a habilidades como la resolución de problemas, el trabajo en equipo y la gestión del tiempo (Hampson, Patton \& Shanks, 2011). En estas tres habilidades está la tecnología como elemento inherente; es así que cabe señalar que existe una fuerte tendencia a implementar las tecnologías en el proceso de aprendizaje-enseñanza por ello a nivel nacional se produce un proceso de transición en la implementación tecnológica, específicamente en lo que se refiere a TIC en Educación, con un diseño curricular establecido en el 2016 y las modificaciones en el 2017, que incluye a la alfabetización tecnológica como una de las competencias transversales a desarrollar en los estudiantes y que se encuentra descrita en mapas de progreso y estándares de aprendizaje a alcanzar en cada uno de sus niveles de la escolaridad.

En este contexto resulta complicado y hasta soñador poder emprender planes de implementación de la TIC integradas a la enseńanza de las áreas del currículo, pues la gran concentración de esfuerzos está en garantizar mínimamente la disponibilidad de dichos recursos en las escuelas, entre otros niveles, siendo uno de los más avanzados la capacidad del docente para planificar unidades de aprendizaje con integración de las TIC. Se sabe que es necesario innovar en la educación peruana, en cuyo contexto se realiza esta investigación, pues las innovaciones son un fuerte potencial para mejorar los resultados en educación y, conscientemente, se sabe que hay mucho por hacer en este sector.

En las nuevas tendencias, la integración de las TIC con fines educativos desempeña un rol fundamental para poder brindar una enseñanza personalizada e individualizada. Adicionalmente a lo anterior, la educación es percibida en muchos países como uno de los medios principales para lograr la equidad, de manera que las innovaciones puedan ayudar a alcanzar este objetivo por lo menos en el acceso a la educación. Ser más eficientes económicamente hablando, es decir, minimizar 
costos y maximizar recursos, también cuenta y cuenta mucho. Este artículo se propuso determinar cuál es la situación actual con respecto a la enseñanza de las Ciencias Naturales a los nativos digitales, como definió Prensky a los estudiantes de este siglo. Se trató también de establecer los niveles de implementación que requeriría la integración de las TIC en la enseñanza y los retos que supone hacerlo, como también algunas propuestas para hacer esto realidad.

\section{MÉTodo}

El presente artículo se realizó a través de la revisión, lectura y análisis de libros, tesis, conferencias virtuales y artículos académicos, haciéndose una búsqueda mediante los descriptores siguientes: Enseñanza de las Ciencias Naturales, integración de los recursos tecnológicos, innovaciones educativas y nuevas Tecnologías de la Información y Comunicación. La búsqueda se realizó en la base de datos de SciELO, Redalyc, Revistas Electrónicas de Investigación Educativa; de Investigación y Experiencias Didácticas; Iberoamericana de Educación en Tecnología y Tecnología en Educación; Actualidades Investigativas en Educación; Revista Cubana de Ciencias Informáticas; de Medios y Educación; The Education Digest y en redes como Flipped Learning Network y Innosight Institute, International Society for Technology in Education (ISTE) y Google Books. Dada la temática del artículo, la información revisada comprende desde 2014 hasta el presente 2017, debido a que abarcan las innovaciones en Educación en materia de integración de las TIC con la enseñanza de las Ciencias Naturales. El estudio examina el impacto generado por algunos de los recursos tecnológicos cuando estos se aplican como un medio para lograr los resultados de aprendizaje esperados en el área curricular de Ciencias Naturales.

\section{La escuela para los estudiantes del siglo XXI}

La escuela del siglo XXI ha pasado de estar centrada en la cobertura del contenido, a la formación en competencias, principalmente. También es posible identificar el cambio de paradigma en que se sustenta, pasando de un paradigma con- ductual hacia la construcción del conocimiento $y$, actualmente, se habla de un paradigma sociocontructivista.

Es en este paradigma que el desarrollo de las competencias cobra real importancia. La competencia, definida como "la capacidad de movilizar varios recursos cognitivos para enfrentarse a una situación” (Perrenoud, 2004), es trabajada en las aulas del siglo XXI a través de situaciones de aprendizaje que, como su definición, exigen en el estudiante procesos de alta demanda cognitiva que integran capacidades y otros recursos con los que cuenta el estudiante, de tal manera que va construyendo su propio aprendizaje en un entorno social del que se nutre y donde, a su vez, aplica lo aprendido. Para que los estudiantes sean competentes se debe trabajar elementos esenciales, entre ellos la articulación sistémica y en tejido de las actitudes, los conocimientos y las habilidades procedimentales dentro de las cuales estarían el manejo de los recursos tecnológicos, el desempeño tanto ante actividades, como en respecto al análisis y resolución de problemas y la idoneidad en el actuar (Tobón et al., 2008).

Los cambios generados a nivel mundial a finales del siglo XIX, con el auge que tuvieron las TIC en todas las esferas y ámbitos, por ejemplo el educativo y el económico, hicieron que el mundo en sí tuviera que cambiar. Esto ocasionó que en las instituciones educativas y otros espacios de aprendizaje coexistan comunidades generacionales diferentes, una de jóvenes y nińos y otra de adultos, que aunque tienen la oportunidad de acceder a los mismos recursos tecnológicos como medio para desarrollar estas habilidades, se diferencien en la funcionalidad que le dan a estos recursos (Cassany, 2008). Entonces se puede hablar también de estas dos generaciones como las denominó Prensky en su momento: los nativos y los inmigrantes digitales.

En la sociedad peruana, los nativos digitales no solo tienen el adicional de la disponibilidad de TIC, sino que además cuentan con un contexto diferente. Y no es que uno sea mejor que el otro, se trata de una cuestión de pertinencia según el contexto. En ese sentido, es necesario considerar que, si los estudiantes están expuestos a diversas 
experiencias, es evidente que sus conexiones neuronales debido a la plasticidad cerebral también serán diferentes, pues dadas las oportunidades de aprendizaje en ellos se establecerán nuevas conexiones que permitirán a las personas desempeñarse en otras habilidades cada más diversas (Garcés y Suárez, 2014). Es por ello importante brindar a los estudiantes diversas oportunidades de aprendizaje para así aprovechar al máximo el tipo de plasticidad que poseen y que depende de la experiencia. Esto cobra aún mayor importancia en estudiantes que tienen trastornos del desarrollo o enfermedades neurodegenerativas (Coll, 2011).

\section{Rol del docente en este siglo XXI}

En la actualidad, los docentes intentan reducir la brecha entre lo que los estudiantes quieren aprender, la forma en cómo quieren aprender y lo que ellos pueden hacer desde su experticia profesional. Así le hacen frente al problema ya documentado por Prensky, quien claramente precisaba que, como educadores de inmigrantes digitales, los docentes hablan una lengua que no es la de los estudiantes. Los docentes pretenden enseñarles un idioma completamente nuevo con el que ellos ya están familiarizados. Es decir, los docentes pretenden enseñar a los estudiantes algo que estos podrían enseñarles mejor a ellos (Prensky, 2001). Por esta razón existe todavía una gran resistencia a la integración de las TIC en la enseñanza por parte de los docentes cuya educación se realizó de forma diferente y no se encuentran familiarizados con las TIC (Sunkel, s/f). Pero también se debe destacar que hay docentes que han comprendido que su rol ha cambiado y se han visto en la necesidad de innovar el proceso de enseñanza utilizando recursos tecnológicos idóneos para apoyar a sus estudiantes; aprovechan las características de plataformas y aulas virtuales de uso libre que permiten la interactividad, la simultaneidad, la retroalimentación entre pares, la generación del inter-aprendizaje. Estas plataformas permiten brindar una diversidad de experiencias educativas y están disponibles y accesibles desde distintos dispositivos, lugares y momentos; constituyen un factor principal para el compromiso que los docentes deben tener para integrar la tecnología en sus unidades de aprendizaje, dado que esa es la forma cómo aprenden los estudian- tes de este siglo (UNESCO, 2013). Conscientes de este nuevo rol, hay docentes que administran evaluaciones en línea, mediante el uso de software que permiten la retroalimentación inmediata, así como el consecuente registro de las calificaciones obtenidas por parte de los estudiantes, generándose mayor motivación en ellos y en el aprendizaje a través de este tipo de actividades.

Si realmente se quiere generar cambios en las aulas, ya se cuenta con el principal agente de cambio: "el estudiante de hoy"; sin embargo, es necesario generar cambios en los docentes. Exponerlos a procesos de formación continua es una manera intuitiva de hacerlo; constituye una estrategia compensatoria en la adquisición de competencias en TIC para aquellos cuya formación dista mucho de la actual (Sunkel, Trucco y Espejo, 2014). La actualización docente considera ahora a docentes que tiendan a trabajar en equipo por el bien de la escuela; entre otras competencias, deberían poder elegir los contenidos pertinentes, emplear la tecnología como recurso del aprendizaje y así estar preparados para un mundo globalizado sometido a cambios constantes. (UNESCO, 2015).

\section{La enseñanza de las Ciencias Naturales en Perú}

Desde el año 2014 se han ido implementando nuevos lineamientos en materia educativa en todo el país; aunque todos son perfectibles como, es normal, sobre todo cuando en materia de currículo se trata, es primordial identificar lo que supone la enseñanza de las Ciencias Naturales. En Perú se cuenta con un currículo nacional que propone tres competencias relacionadas a esta área: (i) "Indaga mediante métodos científicos para construir sus conocimientos, (ii) Explica el mundo físico basándose en conocimientos sobre los seres vivos, materia y energía, biodiversidad, Tierra y universo y (iii) Diseña y construye soluciones" (MINEDU, 2016) estas tres competencias, al igual que las demás estipuladas en las otras áreas curriculares, se deben desarrollar integrando otras dos competencias transversales y enfoques. Una de estas es la que constituye el interés central en este artículo, la referida a desenvolvimientos en entornos virtuales generados por las TIC.

La enseñanza de las Ciencias Naturales exige que 
los estudiantes sean competentes científicamente, es decir, que puedan emplear el conocimiento científico para identificar preguntas y obtener conclusiones basadas en pruebas, con el fin de comprender y poder tomar decisiones sobre el mundo natural y sobre los cambios que la actividad humana produce en este (OCDE, 2000). Lo anterior se debe alcanzar mediante el enfoque de la indagación y la alfabetización científica. Cuando un estudiante aprende ciencias naturales con el enfoque de la indagación y la alfabetización científica, plantea preguntas acerca de un hecho o fenómeno que observa; formula posibles explicaciones sobre dichas observaciones, se involucra en una investigación que previamente diseña, colecta, analiza datos y elabora conclusiones a partir de los resultados que obtiene y su contraste con las explicaciones iniciales (National Science Foundation, 2001).

Actualmente se propone desarrollar en los estudiantes estas competencias a través de las unidades de aprendizaje (MINEDU, 2016), que son secuencias de actividades organizadas en función de una situación problemática que supone que los estudiantes usen capacidades para poderla resolver y en las que la integración de las TIC solo se puede observar en momentos específicos o en proceso de implementación paulatina.

En este punto también es necesario considerar el nivel de infraestructura escolar que se tiene en nuestro país para la enseńanza tanto en el sector privado como en el público. Hay escuelas dotadas de mobiliario escolar y espacios físicos destinados al aprendizaje de las ciencias naturales, la motricidad gruesa y cómputo. Sin embargo, los estudiantes aprenden ciencias naturales y otras áreas del conocimiento de manera integrada, donde el principal soporte son las TIC para poder lograr aprendizajes, no se dispone de aulas donde ellos tengan acceso a diversos recursos incluyendo los tecnológicos como tabletas para organizar su trabajo, aplicaciones tipo simuladores, laboratorios para experimentar las hipótesis que formularon o, mejor aún, buscar información sobre si estas ya fueron comprobadas previamente por otros estudiantes en el mundo, tener la oportunidad de interactuar con otros estudiantes en función de sus dudas, generar debates, compartir puntos de vista.

\section{Las TIC en la Educación}

La integración de las TIC al campo educativo es uno de los objetivos que forma parte de lineamientos nacionales, no solo de nuestro país sino de otros programas como el realizado por Argentina, denominado "Conectar Igualdad" del Ministerio de Educación para la educación media y la formación docente, los desarrollados por el Ministerio de Educación de Colombia como el programa "Computadores para educar", dirigido a la población preescolar, básica, media y superior; el programa "Conexión Total" con el proyecto "Red Educativa Nacional" y el programa de uso de nuevas tecnologías para el desarrollo de competencias, entre otros, que pueden ser visualizadas en el siguiente enlace http://aprende. colombiaaprende.edu.co. Colombia ha tomado seis factores de éxito para integrar las TIC en las escuelas; siendo estos: a) Las infraestructuras físicas y su mantenimiento, b) los recursos educativos digitales, c) la coordinación pedagógica de las TIC, d) la formación y actualización docente para las buenas prácticas TIC del profesorado, e) la integración de las TIC al currículo, f) la integración en los procesos organizativos y de gestión (MEN, 2013).

En Chile también hay experiencias documentadas como las estrategias dirigidas por "Enlaces" Centro de Educación y Tecnología del Ministerio de Educación (http://www.enlaces.cl/) dirigido a la Educación Básica. Brasil es otro de los países que tiene como uno de sus objetivos la implementación de las TIC. Tal es así que experiencias como el programa "UCA", que tenía las bases del Proyecto One Laptop per Child (OLPC), desarrollado por el Laboratorio de Medios del Instituto de tecnología de Massachussets (MediaLab MIT), usa la tecnología móvil, la inclusión digital y social de estudiantes, educadores y comunidad escolar; al uso pedagógico de las TIC (https:// www.media.mit.edu/).

En Perú está la experiencia del proyecto Huascarán del Ministerio de Educación que tuvo un alcance de dos etapas: La primera etapa en educación primaria y la segunda en todos los niveles 
de educación básica y a los docentes. También se debe mencionar el programa "Una Laptop por Niño", en el año 2012, llevado a cabo por la Dirección General de Tecnologías Educativas (DIGETE) del Ministerio de Educación, en el que se les proporcionó laptops $\mathrm{XO}$ a los estudiantes $\mathrm{y}$ docentes de las Instituciones Educativa del nivel Primaria, en todo el país, como una herramienta pedagógica. También está el proyecto TIC para aprender a cargo de la Organización de Estados Iberoamericanos para la Educación, la Ciencia y la Cultura (OEI) en Perú y la Dirección General de Tecnologías Educativas (DIGETE) en el 2014 en Cajamarca, en el nivel primario, para incorporar las TIC y, con ello, apoyar en la mejora de la calidad del proceso de enseñanza aprendizaje (http://educaciontic.perueduca.pe/?p=65).

Los resultados de estos programas son alentadores, como el caso de Brasil, con la implementación del programa "UCA". En medio de lo favorable que eran en cuanto a aprendizajes, existían algunas reflexiones sobre aspectos de la organización curricular, indicios de innovación educativa en la escuela y de cambios en las concepciones, metodologías y prácticas docentes. Pero es importante resaltar que no solo se puede mencionar que es suficiente la incorporación de las computadoras o las TIC, sino que adicionalmente se requieren otros elementos tan necesarios como estas y que van a la par en su proceso de implementación, como los acompañantes pedagógicos o especialistas en el proceso de cambio de las prácticas de enseñanza-aprendizaje de los docentes que implementan las TIC en sus aulas (Alderete, 2016).

\section{La integración de las TIC en la enseñanza de las Ciencias Naturales}

En los diversos niveles educativos, tanto nacionales como internacionales, se hacen múltiples intentos para integrar las TIC al proceso de aprendizaje-enseńanza de las áreas curriculares. Lamentablemente, muchas veces el foco de la atención u objetivo a lograr es dotar de TIC al proceso educativo. La implementación de materiales digitales como tabletas, computadoras, software educativos sin identificar que las TIC no son un fin en sí mismo, sino un empoderador medio para poder alcanzar los resultados de aprendizaje, (UNESCO, 2013). Los modelos de formación que han de implementarse deben responder al cambio constante e imparable que caracteriza a una sociedad del conocimiento y la información en la que vivimos; es decir, no solo se trata de fijarse en una implementación técnica o que las escuelas cuenten con los recursos tecnológicos, sino que estos recursos deben aportar al desarrollo de competencias desde un enfoque pedagógico, didáctico, reflexivo y crítico (Valencia et al., 2016), hoy enunciadas en el currículo nacional y traducidas en capacidades y desempeños.

El criterio principal para integrar las TIC en la enseñanza es que estas tienen un potencial significativo para involucrar a los estudiantes en su proceso educativo, más que tan solo formar parte del repertorio de recursos educativos con los que cuenta un docente (Stone, Franz y Breit, 2005). Desde este punto de vista, y por la experiencia, se puede decir que algo en lo que los docentes muestran interés y despliegue de esfuerzos para alcanzar es en la interactividad de sus prácticas calificadas al final de cada unidad de aprendizaje, hacer repositorios de trabajo de los estudiantes, enviar los recursos didácticos utilizados en clase como las presentaciones con diapositivas, fichas de lectura, entre otros, a través de medios digitales. Por lo tanto, la integración parcial de las TIC a través de esas acciones genera motivación e interés tanto por parte de los estudiantes como del mismo docente aunque las razones de dicha motivación son bastante distantes entre uno y otro agente educativo.

Emplear recursos de realidad aumentada, siendo que esta tecnología o grupo de tecnologías permiten realizar una combinación entre la información real y la información virtual, se convierte en una posibilidad de continuar generando oportunidades de desarrollo de la competencia científica por parte de los estudiantes (Fracchia, De Armiño y Martins, 2015). Por ello, el hecho de que los estudiantes manipulen objetos virtuales, a través de marcadores, como si se tratasen de objetos reales, tales como los generados por recursos libres y al alcance de estudiantes, docentes e investigadores, como lo son: Geogebra (https://www.geogebra. org/?lang=es), Scratch (https://scratch.mit.edu/), Kodu (https://www.kodugamelab.com/), Mind- 
craft (https://minecraft.net/es-es/) y los de realidad aumentada o 3D (https://human.biodigital. com), permiten que el estudiante realice operaciones de forma natural e intuitiva, sin necesidad de un soporte técnico diferente al que usualmente tienen; esto también es un novedoso e innovador medio de enseñanza de las Ciencias Naturales y de las demás áreas curriculares. La tecnología aplicada al campo de la enseńanza de las Ciencias Naturales genera significatividad a los aprendizajes construidos por los estudiantes, cuando esta es usada como un medio. La tecnología es un medio clave para que los estudiantes trabajen en sus concepciones alternativas. Esas explicaciones intuitivas sobre el mundo que los rodea, pero que tienen que pasar por un cambio conceptual para constituirse en una forma pertinente y científica de explicar aquello que les acontece y sobre lo que reflexionan en su día a día.

Otra de las estrategias docentes mayormente usada a lo largo de los tiempos para enseñar ciencias naturales es el laboratorio. En la actualidad existe la oportunidad de llevar a acabo laboratorios virtuales y recursos que permiten realizar el procesamiento con el consecuente análisis de datos obtenidos a partir de dichas experimentaciones virtuales y que se constituye en una aproximación bastante acertada al tan ansiado cambio conceptual por parte de los estudiantes. Existe evidencia sobre la efectividad de la estrategia de los laboratorios virtuales para trabajar la propuesta didáctica referida al estudio de la termodinámica. Se aplicó un instrumento tecnológico que permitía la detección y colección de datos en tiempo real lo cual mejoro considerablemente (hasta en un $400 \%)$ la distinción entre calor y temperatura por parte de los estudiantes. Por ello es relevante enunciar aquellos simuladores gratuitos que se tienen para la enseñanza de las ciencias, como el proyecto $\mathrm{PhET}$ Interactive Simulations de la Universidad de Colorado Boulder (https://phet. colorado.edu/), el cual ofrece simulaciones interactivas gratuitas de matemáticas y ciencias que, en un ambiente intuitivo y lúdico, están orientadas a involucrar a los estudiantes en su proceso de aprendizaje a través de la exploración y el descubrimiento. El the Molecular Workbench (http://mw.concord.org/nextgen/), proporciona simuladores para enseñar y aprender ciencia. El
Jet Propulsion Laboratory California Institute of Technology (http://space.jpl.nasa.gov/) brinda a la oportunidad de colocar parámetros deseados para acceder a vistas y simulaciones sobre los cuerpos en el Universo.

Actualmente se habla de integrar estas herramientas tecnológicas en espacios virtuales donde los estudiantes tengan acceso no solo a ellas y a otras actividades que el docente puede programar como una evaluación en línea sobre los aprendizajes generados mediante el uso de un simulador, un video, participar en un debate sobre alguna cuestión socio-científica, acceder a otros recursos en formatos diferentes o llevar el control de sus propios avances y con ello gestionar su propio aprendizaje.

La diversidad de implementación de plataformas virtuales para el aprendizaje de las Ciencias $\mathrm{Na}$ turales está permitiendo también que estas sean utilizadas y apoyen el proceso de aprendizaje- enseñanza, como es el Entorno Web de Indagación en Ciencias, creado por un equipo de la Universidad de Berkeley, California, que ofrecen recursos TIC para integrar secuencias didácticas con herramientas de gestión de clase y de evaluación (Irribaren, Furman, Podestá y Luzuriaga, 2014).

El hecho de que los estudiantes accedan a estos recursos TIC como videos, animaciones, simuladores, gráficos dinámicos que generan oportunidades para que los estudiantes puedan plantear hipótesis; diseñar, realizar experimentos y justificar sus respuestas se consolida como una herramienta en conjunto, potente para generar competencia científica y actitudes favorables tanto por parte de los estudiantes como de los otros integrantes de la comunidad educativa, como los propios padres de familia, docentes, pares, líderes de la escuela.

Una de las plataformas que ha permitido ser un soporte para los materiales de clases de ciencias naturales es la implementación del Moodle (https://moodle.org/?lang=es). Este es un espacio virtual de aprendizaje para los estudiantes y de esta forma lo que plantean es la metodología Blended learning o B- Learning, es decir, espacios de aprendizaje en un aula física con otros aprendizajes que se generan por intermedio de un aula virtual. Dentro de la Educación Básica regu- 
lar encontramos también experiencias exitosas de su implementación, como en la institución educativa Los Ángeles de España (http://plataforma.ieslosangeles.es/course/category.php?id=3).

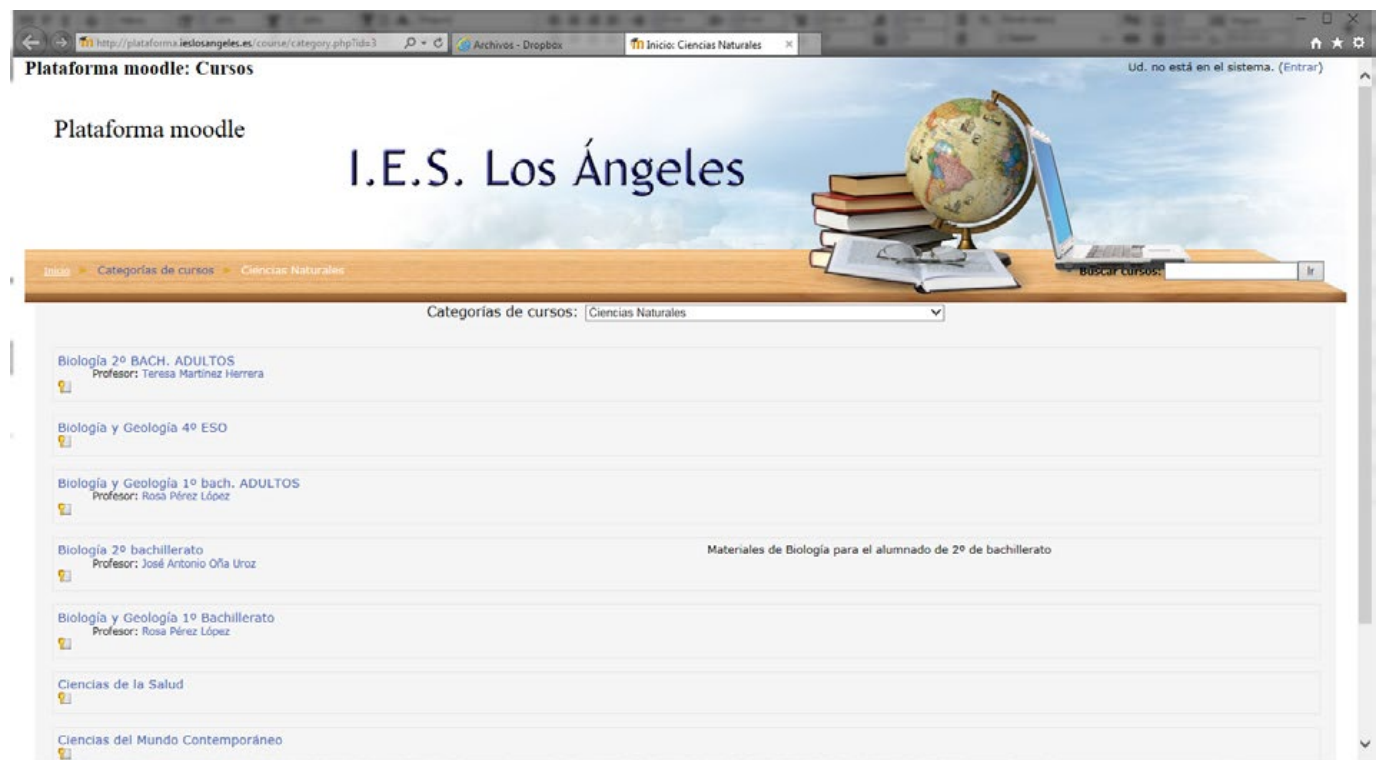

Figura 1. Plataforma Moodle de Ciencias Naturales.

Fuente: http://plataforma.ieslosangeles.es/course/category.php?id=3

Otro de estos entornos virtuales, la plataforma Edmodo, que es gratuita, permite al docente organizar sus actividades por grupos, diversificarlas, enlazarlas con otras webs, integrar aplicaciones, evaluaciones en línea, encuestas, foros y obtener los resultados para comparar y establecer planes de mejora. Edmodo funciona de un modo análogo a Facebook, pues permite que el docente pueda invitar a sus estudiantes a grupos que él previamente ha organizado; colocar fotos de actividades compartidas; dar "me gusta", comentar; participar en una temática de discusión, con el valor agregado de dar a los padres acceso para que conozcan en qué invierten el tiempo sus hijos(as), tanto a nivel de comentarios en plataforma como en el de evaluaciones calificadas, pues la plataforma proporciona un dashboard actualizado en tiempo real. Por otra parte, el docente tiene el control de los estudiantes que acceden al grupo, cómo lo hacen y la posibilidad de asignarles roles.

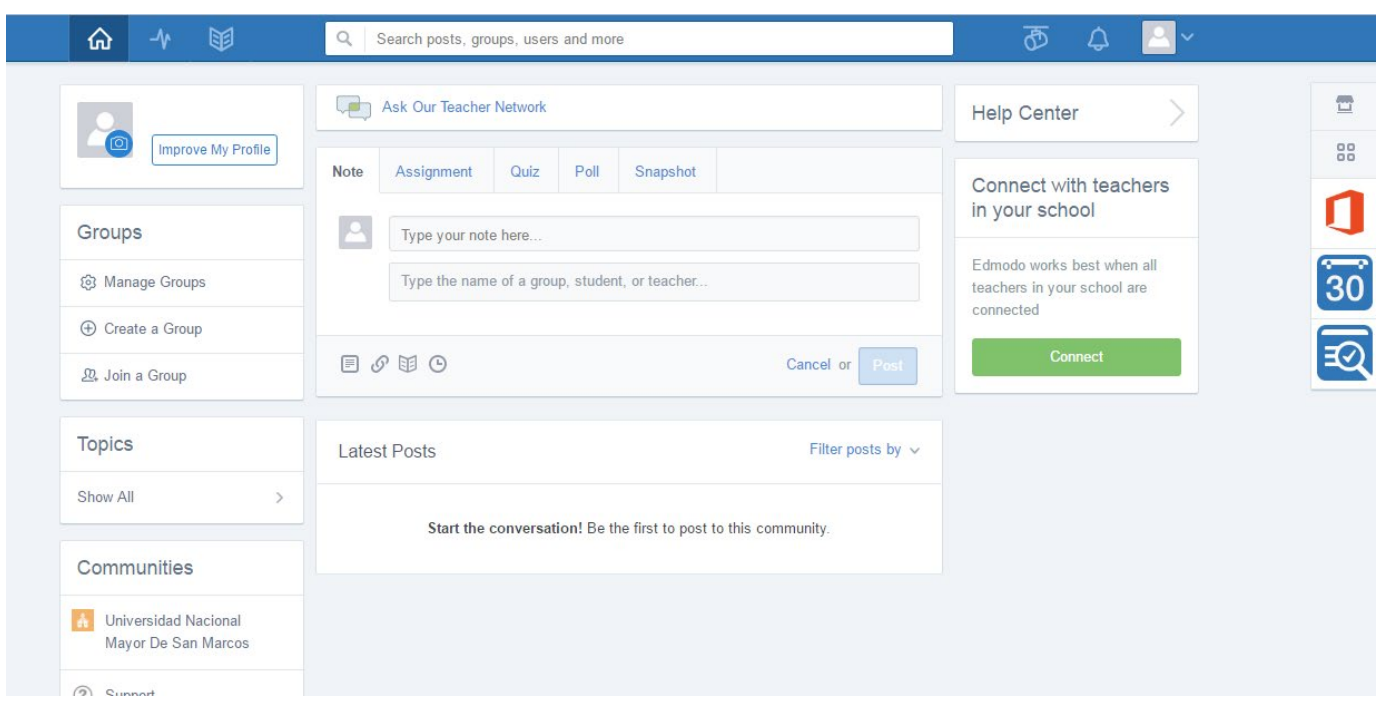

Figura 2. Inicio en la plataforma de edmodo.

Fuente: https://www.edmodo.com/?language=es 
A través de la implementación de este tipo de estrategias se ha documentado que los estudiantes pueden construir aprendizajes significativos, pues favorece la motivación hacia la participación: propicia el trabajo colaborativo e incrementa el interés de los estudiantes en situaciones de aprendizaje que se trabajan (Cuenca, 2015).

Es posible apoyar todos los contenidos curriculares usando las TIC. El éxito de esta implementación depende de la mediación pedagógica de los docentes, sus conocimientos y formas de gestionar el aprendizaje de sus estudiantes con dichos recursos (Sunkel, s.f). Estos pueden ser modelos de enseñanza que contemplen el "blended learning”, aprendizaje basado en proyectos y ambientes personalizados de aprendizaje, harán posible la incorporación educativa de juegos, redes sociales, de plataformas en línea, de videos y otros recursos tecnológicos, incluso a estudiantes de menores recursos económicos (UNESCO, 2013).

\section{CONCLUSIONES}

La integración de las TIC es un medio importante para generar aprendizajes en los estudiantes en lo que respecta a Ciencias Naturales. Existe diversidad de recursos tecnológicos, como por ejemplo los destinados a la organización y el procesamiento de datos como el uso de simuladores: recursos que desarrollan y/o refuerzan las capacidades que integran las competencias de esta área siendo una de las principales las de "Diseñar estrategias para hacer indagación" y "Generar y registrar datos o información”.

Para poder integrar las TIC a la enseñanza no solo de las Ciencias Naturales, sino de cualquier otra área curricular se puede realizar en diversos niveles considerando primordialmente la preparación de los agentes educativos (estudiantes, padres de familia, docentes y líderes de las escuelas) tanto a nivel cognitivo como actitudinal, y los recursos con los que se cuentan para acceder a la tecnología que se desea implementar siendo el objetivo, la mejora de la calidad de los aprendizajes en el área en el que se están implementando y, mejor aún, si se busca esta mejora de manera inter-áreas, en proyectos que integren las diversas áreas curriculares. Estos niveles de implementación pueden ser paulatinos, a través del uso de recursos que potencien secciones específicas de las unidades de aprendizaje o utilizando metodología como el Blended Learning o Flipped the Class, metodologías en los que la inclusión de la tecnología goza de un espacio privilegiado en tanto que hace que el momento de enseńanza de una determinada área vaya más allá del aula y en espacios gestionados por el estudiante y guiados por el docente sin que ambos estén físicamente presentes.

Se debe considerar que dentro de los retos que demanda la integración de las TIC a la enseñanza de las Ciencias Naturales, está el que los estudiantes no estén dispuestos a realizar la inversión de esfuerzo que conlleve un modo de aprender distinto al que generalmente están acostumbrados. Sin embargo se debe de tener como norte que la integración de las TIC es uno de los medios necesarios para alcanzar los resultados de aprendizaje que se espera en los estudiantes.

Además, en los entornos virtuales atractivos pero inestables, los estudiantes están comprometidos y poseen estrategias de aprendizaje en las que las plataformas virtuales se constituyen como medios privilegiados para proponer tareas que involucran participación entre pares, como por ejemplo los foros y debates tienen mayores tasas de cumplimiento que aquellas que requieren de trabajo individual. Sin embargo, la participación de los estudiantes se aleja de los criterios de calidad que el docente espera por más que estos sean establecidos como reglas de juegos, desde el inicio de la actividad. Pero evidentemente es un proceso continuo de formación, por ello la solución no está en dejar de lado la integración de las TIC al proceso de aprendizaje-enseńanza, sino que se debe ir aprendiendo en el camino, generando estrategias innovadoras.

La integración exitosa de las TIC tiene como componente central luego del actuar de los estudiantes, el de los docentes, que necesitan condiciones propicias para desempeñarse en dicha integración y capacitaciones organizadas de la misma forma, en cómo se espera que ellos se apropien de estas herramientas tecnológicas en el aula de clases. 
Otro agente sumamente importante son los padres de familia, quienes demandan la integración de las TIC, pero necesitan mayor información de lo que esto significa o significará de su parte, pues para que la integración de las TIC en la enseñanza de las ciencias naturales y, en general, de toda área, es necesario que los padres de familia estén involucrados, ya sea guiando y/o monitoreando el desempeño académico de sus hijos.

Se debe tener en cuenta que, si bien es cierto, existen materiales gratuitos, sin mayores requerimientos de software; estos no siempre están alineados al desarrollo de competencias, por lo que se deben de producir en "in house", adaptarse también es una buena opción.

\section{REFERENCIAS BIBLIOGRÁFICAS}

Alderete, M., y Formichella., M. (2016). Efecto de las tic en el rendimiento educativo: el Programa Conectar Igualdad en la Argentina. Revista Cpal. Recuperado de: https://doi. org/10.18356/c7045fd1-es.

Bergmann, J., y Sams, A. (2012). Flipped yor classroom: Reach every student in every classevery day. EEUU: International Society for Technology in Education.

Bruni, J., Aguirre, L., Díaz, H., Fernandez, A. y Barrios. M. (2008). Una mejor educación para una mejor sociedad. Propuestas para el diálogo y la transformación educativa en en América Latina y el Caribe. Federación Internacional de Fe y Alegría.

Cajiao, F. (2001). La sociedad educadora. 26. Organización de los Estados Iberoamericanos.

Cassany, D (2008). Nativos e inmigrantes digitales en la escuela. Educativa, Ed. Barcelona: Estudios e Investigaciones.

Coll, A. (2011). XII Congreso Internacional de Teoría de la Educación. Plasticidad cerebral y experiencia:fundamentos neurobiológicos de la educación. Barcelona: Dept. Psicobiologia i Metodologia de les Ciències de la Salut. Institut de Neurociències. Universitat Autònoma de Barcelona.

Coll, C. (s.f). Los desafíos de las TIC para el cambio educativo. Aprender y enseñar con las TIC:expectativas, realidad y potencialidades. Espańa: Organización de Estados Iberoamericanos para la Educación.

Cuenca, C. (2015). El foro virtual como estrategia de enseñanza en la educación superior. Hamut'ay, 2 (1) 23-31. Recuperado de: https://doi.org/10.21503/hamu.v2i1.827

Fernández, A. (2016). Modelo educativo emergente en las buenas practicas TIC. 1(18), 33-47. Recuperado de: https:// doi.org/10.12795/revistafuentes.2016.18.1.02
Fracchia, C., De armińo, A., y Martins, A. (2015). Realidad Aumentada aplicada a la enseńanza de las Ciencias Naturales. Revista Iberoamericana de Educación en Tecnología y Tecnología en Educación (16), 7-15.

Fulton, K. (2012). Upside Down and Inside Out: Flip Your Classroom to Improve Student Learning. Learning \& Leading with Technology. International Society for Technology in Education.

Garcés, M., y Suárez, J. (2014). Neuroplasticidad: aspectos bioquímicos y neurofisiológicos. Revista Ces medicina, 28(1).

Hampson M., Patton A. \& Shanks L. (2011). 10 schools for the 21 st century. Innovation Unit.

Irribaren, R., Furman, M., Podestá, M. y Luzuriaga, M. (2014). Diseño e implementación de la plataforma virtual de aprendizaje WISE en el aprendizaje de las Ciencias $\mathrm{Na}$ turales. Congreso Iberoamericano de Ciencia, Tecnología e Innovación en Educación.Argentina.

Marticorena, B. (Agosto de 2006). Tecnología y Educación. 7(2). Perú: Editorial Norma.

Ministerio de Educación Nacional de Colombia. (2013). Competencias TIC para el Desarrollo Profesional Docente. Obtenido de http://www.colombiaaprende.edu.co/html/ micrositios/1752/articles-318264_recurso_tic.pdf

Ministerio de Educación del Perú. (2016). Currículo Nacional de la Educación Básica.Obtenido de http://www.minedu.gob.pe/curriculo/pdf/curriculo-nacional-2016-2.pdf.

National Science Foundation. (2001). A Monograph for Professionals in Science, Mathematics, and Technology Education: Professional Development that Supports School Mathematics Reform. 3.

Organización para la Cooperación y el Desarrollo Económicos. (2000). Measuring Student Knowledge and Skills: A new Framework for Assessment. Paris.

Prensky, M. (diciembre de 2001). Nativos Digitales, Inmigrantes Digitales. MCB University Press, 9(6).

Proyecto the flipped classroom. (2012). Un infográfico sobre Flipped Classroom. España.

Revista Electrónica de Investigación Educativa. Recuperado de: http://redie.uabc.mx/redie/issue/archive

Revista de Investigación y Experiencias Didácticas. Recuperado de: http://ensciencias.uab.es/index

Revista Iberoamericana de Educación en Tecnología y Tecnología en Educación. Recuperado de: http://teyet-revista. info.unlp.edu.ar/

Revista Electrónica Actualidades Investigativas en Educación. Recuperado de: http://revistas.ucr.ac.cr/index.php/aie Revista Cubana de Ciencias Informáticas. Recuperado de: http://rcci.uci.cu/?journal=rcci\&page=issue\&op=archive

Revista de Medios y Educación. Recuperado de: http://acdc. sav.us.es

Sams, A., y Bergmann, J. (2012). Flip your Classroom Reach every student in every class every day. EEUU: International Society for Technology in Education. 
Schalk, Q. (2010). El impacto de las TIC en la educación. Brasil: Organización de las Naciones Unidas para el desarrollo de la Educación, la Ciencia y la Cultura.

Staker, H., y Horn, M. (2012). Classifying K-12 Blended Learning. Innosight Institute.

Stavert, B. (2013). Bring Your Own Device (BYOD) in Schools: Literature Review. (D. o. Communities, Ed.) Technology for Learning Program - Information Technology Directorate.

Stone, W., Franz, R. y Breit, L. (2005). Teaching for understanding eith technology. EEUU: Jossey Bass.

Sunkel, G. (s.f). Las nuevas tecnologías de la comunicación y la información (TIC) en la Educación. Desafíos para las políticas públicas en América Latina. Comisión Económica para América Latina y el Caribe.

Sunkel, G., Trucco, D., y Espejo, A.(2014). La integración de las tecnologías digitales en las escuelas de América Latina y el Caribe: Una mirada multidimensional. Chile : Comisión Económica para América Latina y el Caribe (CEPAL).

Thomas, R. \& Tighe, J. (2003). Backward Desing for Forward Action. 60(5).

Tobón, T., García, F., Rodriguez, S., y Lopez, R. (2008). Gestión del Currículo por Competencias: Una aproximación desde el modelo sistémico complejo. Perú: AB Representaciones Generales S.R.L.

Organización de las Naciones Unidas para la Educación, la Ciencia y la Cultura. (2013). Enfoques estratégicos sobre las TIC en Educación en América Latina y el Caribe. Chile: Organización de las Naciones Unidas para la Educación, la Ciencia y la Cultura.

Organización de las Naciones Unidas para la Educación, la Ciencia y la Cultura. (2015). Replantear la Educación ¿Hacia un bien común mundial? Francia : Organización de las Naciones Unidas para la Educación, la Ciencia y la Cultura.

Perrenoud, P. (2004). Diez nuevas competencias clave para enseñar. Barcelona: Graó.

Upside Down and Inside Out: Flip Your Classroom to Improve Student Learning. (s.f.). Learning \& Leading with Technology. International Society for Technology in Education.

Valencia, T., Serna, A., Ochoa, S., Caicedo, A., Montés, J., y Chávez, J. (2016). ICT Standards competences from the pedagogical dimension: A perspective from levels of ICT adoption in teachers' education practice. Colombia: Pontifica Universidad Javeriana. 